\title{
Some tricks for ameliorating the trace-conditioning deficit
}

\author{
ROBERT C. BOLLES, ALEXIS C. COLLIER, MARK E. BOUTON, \\ and NANCY A. MARLIN \\ University of Washington, Seattle, Washington 98195
}

\begin{abstract}
Trace procedures, with a gap between the conditioned stimulus (CS) and the unconditioned stimulus (US), often produce weaker conditioning than procedures with contiguous CS and US. Such deficits were found in this experiment using rat subjects and conditioned suppression techniques to assess the strength of fear conditioning. But the deficit was greatly reduced either by filling the CS-US gap with a second "filler" stimulus or by adding a brief "safety signal" at the start of the intertrial interval.
\end{abstract}

It was Pavlov (1927) who first noted that conditioning was possible with what he termed a trace procedure, in which the conditioned stimulus (CS) is presented only briefly and is not contiguous with the unconditioned stimulus (US). Pavlov observed that trace procedures were not particularly effective, and subsequent experimenters confirmed this observation. For example, Davitz, Mason, Mowrer, and Viek (1957) and Kamin (1961) reported that CS-US gaps of just a few seconds seriously impaired fear conditioning. Kamin (1954) and Mowrer and Lamoreaux (1951) reported severe decrements in avoidance learning with short gaps between the avoidance "CS" and shock. Although conditioning decrements are not always found (e.g., Brahlek, 1968), they do seem to occur under a wide variety of conditions.

The present studies examine some procedures for eliminating or reducing the trace-conditioning deficit. These particular procedures were suggested by Mowrer and Lamoreaux's (1951) hypothesis that the deficit results from the animal's failure to discriminate the interstimulus interval from the intertrial interval. If the animal confuses the short, dangerous "silent" part of the interstimulus interval with the long, safe silence of the intertrial interval, then it should tend not to respond during the former and to respond too much during the latter (a result that Mowrer and Lamoreaux found). It also follows that the conditioning deficit should be ameliorated by marking or designating in some way one silent period or the other so that they are not so likely to be confused. We examined two ways of doing this; first, we tried to mark the short interstimulus interval, then we attempted to mark the long intertrial interval. Both approaches were successful.

This research was supported by Grant GB-40314 from the National Science Foundation. Ms. Collier is now at the Ohio State University; Ms. Marlin is at Brooklyn College of the City University of New York. Reprint requests should be addressed to R. C. Bolles, Department of Psychology, University of Washington, Seattle, Washington 98195.

\section{EXPERIMENT 1}

Our first approach to the problem was to fill the gap in the interstimulus interval with a filler, a second CS, to make the CS-US gap discriminably different from the intertrial interval.

\section{Method}

Subjects. The animals were 36 naive female rats, of Wistar descent, with initial body weight of approximately $300 \mathrm{~g}$.

Apparatus. The animals were trained and tested in Foringer Skinner boxes. Fear conditioning was carried out in wooden boxes of approximately the same dimensions, with the same type of grid floor, and illumination and masking noise (exhaust fan) conditions. Shock was scrambled, of .5 -sec duration and 1-mA intensity. The CS was a white noise of approximately $75 \mathrm{~dB}$ in both apparatuses, and the additional stimulus was a 3,300-Hz tone, also about $75 \mathrm{~dB}$.

Procedure. The animals were reduced to and maintained at $80 \%$ of initial body weight. They were trained to barpress for food on a variable-interval (VI) 90 -sec schedule. This took 5 days.

Half of the animals then received one session of classical conditioning; the other half received four sessions. There were 16 CS-US pairings, averaging every $3.5 \mathrm{~min}$, in each 1 -h session. Each half of the animals was randomly divided into three groups, which differed in the kind of classical conditioning procedure that was used. The delay groups had a 30.5 -sec CS that terminated with the US. The trace groups had a $25-\sec$ CS, followed by $5 \mathrm{sec}$ of silence, followed by onset of the US. The sequential, or filler, group had the same $25-\mathrm{sec}$ CS, followed by $5.5 \mathrm{sec}$ of the $3,300-\mathrm{Hz}$ tone, the last $.5 \mathrm{sec}$ of which overlapped the US. All groups were returned to the Skinner boxes on the baseline task for 1 day to recapture the response baseline and for testing on the last day.

Testing consisted of four probes of the CS alone for $30 \mathrm{sec}$ every $6 \mathrm{~min}$ during the 30 -min test session. The effectiveness of different conditioning treatments was assessed in terms of the suppression of barpressing below baseline level in response to the probed CS. Suppression was indexed by the customary $A \div A+B$ ) formula, where $A$ is the response rate (in responses per minute) during $C S$ presentation and $B$ is the response rate per minute during the $30 \mathrm{sec}$ preceding CS presentation. The suppression ratios were averaged over the four test probes to give each animal a summary suppression ratio (SR). 


\section{Results}

An analysis of variance (ANOVA) of the SR scores was carried out using an orthogonal-comparisons technique (Edwards, 1972). The analysis revealed a reliable trace-conditioning deficit between the 16-delay and 16-trace groups $[F(1,30)=7.88, p<.01]$, but no comparable trace deficit among the 64-pairing groups $[F(1,30)=1.91, p>.10]$. None of the other comparisons (overall 16 vs. 64 pairings, or filler vs. delay and trace) was significant. In other words, there was a trace decrement among the 16-pairing groups but not among the 64-pairing groups.

Other indices of suppression were calculated and are reported in Table 1 . The second column gives the mean SR, calculated in the usual manner but for the response rate during the $30-\mathrm{sec}$ period immediately following CS offset, relative to the pre-CS rate values. These values closely mirror those in the first column. Indeed, the correlation across animals between the two suppression indices was .78. The third column assesses loss of baseline. It presents suppression ratios based on the response rate over all non-CS periods after the first relative to the first non-CS period, before the first CS was presented. Moderate loss of baseline was shown in most groups and, although group differences were generally not reliable, individual differences were. Thus, the correlation across animals of this measure with CS suppression ratios was .64. In short, those animals which were most suppressed by the CS also tended to show the greatest loss of baseline during non-CS periods.

The critical experimental question is whether, among the 16-pairing groups, the .22 of the filler group is more like the .09 of the delay group or the .40 of the trace group. Alas, we know of no direct statistical test of such a question. But there is indirect evidence: $t$ tests showed the .22 but not the .40 to be different from .50 ; the four animals giving no evidence of any suppression on any probe test were all in the 16-trace group; the 16-trace and 16-filler groups differed reliably $(\mathrm{t}=2.8)$ in mean suppression on the first probe test. This type of evidence indicates that both Group 16-filler and Group 16-delay showed reliably more suppression that Group 16-trace, and therefore supports the conclusion that the filler condition does ameliorate the trace-conditioning deficit.

Table 1

Mean Suppression Ratios for the Different Groups in Experiment 1

\begin{tabular}{ccccc}
\hline & & CS & Post CS & Baseline \\
\hline \multirow{4}{*}{ 16 Pairings } & Delay & .09 & .13 & .28 \\
& Trace & .40 & .36 & .53 \\
& Filler & .22 & .22 & .39 \\
\multirow{4}{*}{64 Pairings } & Delay & .24 & .40 & .38 \\
& Trace & .39 & .40 & .43 \\
& Filler & .28 & .36 & .45 \\
\hline
\end{tabular}

Note-See text for a description of the different indices of suppression.

\section{EXPERIMENT 2}

The purpose of this small study was to tie up some loose ends. It involved two filler groups with the stimuli counterbalanced and with suppression tests of both the CS and the filler.

\section{Method}

Twelve animals, from the population described before, were run in the same apparatus and under the same conditions, except as noted. The animals were randomly assigned to two groups: In the conditioning phase, the noise-tone group received a $25-\mathrm{sec}$ white noise CS, immediately followed by a $5.5-\mathrm{sec}$ tone filler, which was conterminous with the $.5-\mathrm{sec}$ shock. Group tone-noise had the tone for the CS and the white noise for the filler. In the test phase, tone and white noise were presented alternately in $30-\mathrm{sec}$ probes every $6 \mathrm{~min}$; the groups were split into subgroups, one of which was probed with the tone first, the other with the noise first. There were two test sessions.

\section{Results}

An ANOVA of the mean SRs revealed no overall difference between the tone and the white noise, between CS and filler, or between the first-presented and second-presented test stimulus (all Fs $<1$ ). The overall mean SR to the CS was .25 the first day (.21 for the filler) as against .22 in Experiment 1. And there was a correlation of .80 across animals between suppression ratios for the CS and for the filler. Trend tests over the four tests for each stimulus indicated no significant effects or interactions not subsumed by the overall linear trend $[F(1,80)=11.3, p<.01]$. This trend merely reflects the loss of suppression typical of the off-baseline procedure; the mean SR ranged from .18 on the first probe test to .37 on the last one.

\section{EXPERIMENT 3}

Now we turn to the question of whether it is possible to mark the long intertrial interval so that it is made discriminately different from the short interstimulus gap. We reasoned that probably only the first part of the intertrial interval is likely to produce confusion and that a quite short additional stimulus might suffice to distinguish the beginning of the intertrial interval from the interstimulus interval. A short cue following shock might also be able to serve as a safety signal (LoLordo, 1969), evoking quite different behaviors from those elicited by the CS, and this mechanism might provide a further basis for the animal to distinguish between the interstimulus and intertrial intervals.

\section{Method}

Eighteen animals of the same description were tested as before in the same apparatus, but with the following differences in procedure. First, all animals were trained and tested in the dark. The two 6-W lamps in the Skinner box were used as one cue. Second, each group of six animals was split into two subgroups to counterbalance light onset and white noise as the CS 


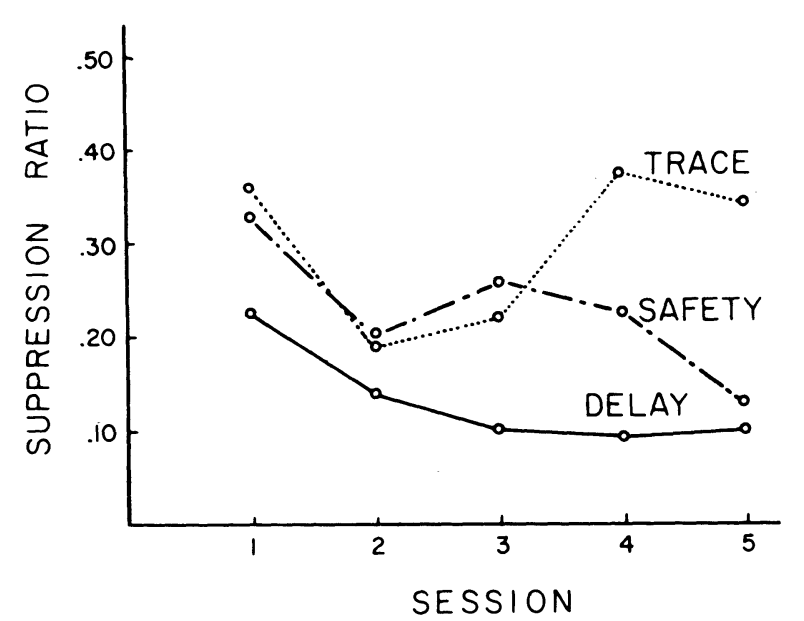

Figure 1. Development over sessions of suppression in the different groups in Experiment 3.

and the safety signal. Third, conditioning was carried out on the baseline task, in the Skinner box, with 20 CS-US pairings per session for five sessions. A somewhat longer interval, $5 \mathrm{~min}$, was used between conditioning trials, and the US was extended to $1 \mathrm{sec}$. There were three groups of animals. The delay group received a $20-\sec$ CS (either light or noise) which ended with US onset. The trace group received a 10-sec CS, followed in $10 \mathrm{sec}$ by the US. The safety group also received a 10 -sec CS, followed in $10 \mathrm{sec}$ by the US, but the US was immediately followed by a 5 -sec safety signal (either noise or light).

\section{Results}

The development of suppression to the CS over the five sessions for the three groups is shown in Figure 1. An ANOVA of the first day's SR data revealed no significant differences apart from a large stimulus effect: The light produced more suppression than the noise $[F(1,12)=4.83, p<.05]$. In the early sessions, the trace deficit was not reliable. Thus, with numbers of pairings comparable to those given in Experiment 1, the pattern of group differences is quite different.

In the later sessions, group differences began to emerge. In the fifth session, while there was still an overall stimulus effect $[F(1,12)=15.6, p<.01]$, there was also a groups effect $(F=8.6)$, and no interaction $(F=2.65)$. Pursuing the groups effect with tests of individual degrees of freedom showed reliably more suppression in both the safety and the delay groups than in the trace group. So, by the fifth session the data indicate that adding a short safety signal at the beginning of the long intertrial interval can ameliorate the traceconditioning deficit. An ad hoc repeated-measures ANOVA on just the data for the trace group indicated that the quadratic trend was reliable $[F(1,20)=4.87$, $\mathrm{p}<.05]$; neither the linear trend nor the remainder was reliable. Evidently, suppression in the trace group gradually increased and then gradually declined. The decline might be attributed to inhibition of delay (Brahlek, 1968), except for the fact that there was no comparable decline in the safety group (Figure 1).

\section{DISCUSSION}

The results of Experiment 1 show that 64 pairings of $\mathrm{CS}$ and US produce quite different effects when the CS is later probed on an appetitive baseline than 16 pairings. We might presume that with the more extended conditioning experience the rat has more opportunity to make some subtle discriminations. Perhaps animals under the delay conditioning were beginning to demonstrate inhibition of delay, perhaps with the extended conditioning experience adaptation to the US had begun to occur, or perhaps the animals were better able to discriminate the test situation from the conditioning situation. Whatever the case, the groups with more conditioning trials showed generally less, rather than more, suppression in the test situation, and there were no reliable differences between groups.

Experiment 1 also presents the interesting finding that suppression is rather general in the sense that those animals whose responding was most suppressed by the CS also tended to have the most depressed baseline. Perhaps this effect is due to individual differences in the persistence of freezing or to the kind of behavior being elicited by the CS to compete with barpressing. One implication of this finding is that, whereas the customary SR may provide a good measure of the fear conditioned specifically to the CS, it may be a poor measure of how much fear there is in the test situation.

The chief finding in Experiment 1 was that a traceconditioning decrement, relative to delay conditioning, was found. And it was found to be significantly reduced by the simple expedient of adding a second CS, or filler, to fill the silent gap between CS and US. Further speculation about the mechanism underlying this ameliorating effect is inappropriate at this time. But the results of Experiment 2 indicate that the effect is not tied to special properties of the stimuli used in Experiment 1; it has some generality over stimulus dimensions. Experiment 2 also showed that the CS and the filler undergo very comparable amounts of fear conditioning.

The principal finding in Experiment 3 was that a short cue added to the intertrial interval immediately after delivery of the US tended to ameliorate the trace-conditioning deficit. Our data suggest, however, that this effect may only appear after more extended conditioning. In contrast with the results of Experiment 1 , in which the trace deficit disappeared with extended conditioning, and the ameliorating effect with it, neither effect was reliable in Experiment 3 until the animals had received 80 conditioning trials. We cannot tell at this point whether this contrary dependence upon amount of conditioning should be attributed to the use of on-baseline vs. off-baseline procedures to index conditioning or whether it is inherent in the discriminabilities of the short interstimulus interval and the long intertrial interval. Perhaps the difference is related to the principle that safety signals are more slowly established with conditioning procedures than are danger signals (Bolles, 1972). The slow onset of the ameliorating effect in Experiment 3 is certainly consistent with the idea that the effect is partly due to the safety signal properties of the added stimulus.

All of these results appear to be consistent with Mowrer and Lamoreaux's (1951) proposal that the trace-conditioning deficit results from the animal's confusion of the interstimulus gap with the intertrial interval, and the idea that such confusion can be reduced by marking one or the other of these non-CS periods with some kind of additional cue.

\section{REFERENCES}

Bolles, R. C. The avoidance learning problem. In G. H. Bower (Ed.), The psychology of learning and motivation (Vol. 6). New York: Academic Press. 1972. 
Brahlek, J. A. Conditioned suppression as a function of the number of stimuli that precede shock. Psychonomic Science, 1968, 12, 189-190.

Davitz, J. R., Mason, D. J., Mowrer, O. H., \& Viek, P. Conditioning of fear: A function of the delay of reinforcement. American Journal of Psychology, 1957, 70, 69-74.

EDWARDs, A. L. Experimental design in psychological research (4th ed). New York: Holt, Rinehart, \& Winston, 1972.

Kamin, L. J. Traumatic avoidance learning: The effects of CS-US interval with a trace-conditioning procedure. Journal of Comparative and Physiological Psychology, 1954, 47, 65-72.
Kamin, L. J. Trace conditioning of the conditioned emotional reaction. Journal of Comparative and Physiological Psychology, 1961, 54, 149-153.

LoLoRdo, V. M. Positive conditioned reinforcement from aversive situations. Psychological Bulletin, 1969, 72, 193-203.

Mowrer, O. H., \& Lamoreaux, R. R. Conditioning and conditionality (discrimination). Psychological Review, 1951, 58, 196-212.

Paviov, I. P. Conditioned reflexes. London: Oxford University Press, 1927.

(Received for publication February 24, 1978.) 